\title{
Exact solution of the Schwarzian theory
}

\author{
Vladimir V. Belokurov* \\ Lomonosov Moscow State University, Leninskie gory 1, Moscow, 119991, \\ Russia and Institute for Nuclear Research of the Russian Academy of Sciences, \\ 60th October Anniversary Prospect 7a, Moscow, 117312, Russia \\ Evgeniy T. Shavgulidz母 \\ Lomonosov Moscow State University, Leninskie gory 1, Moscow, 119991, Russia
}

The explicit evaluation of the partition function in the Schwarzian theory is presented.

The Schwarzian theory [1] is the basic element of various physical models including the SYK model and the two-dimensional dilaton gravity (see, e.g., 2], 3], [4], [5], and references therein). The action of the theory is

$$
I=-\frac{1}{g^{2}} \int_{0}^{2 \pi}\left[\mathcal{S}_{\phi}(\tau)+\frac{1}{2}\left(\phi^{\prime}\right)^{2}(\tau)\right] d \tau .
$$

Here,

$$
\mathcal{S}_{\phi}(t) \equiv\left(\frac{\phi^{\prime \prime}(t)}{\phi^{\prime}(t)}\right)^{\prime}-\frac{1}{2}\left(\frac{\phi^{\prime \prime}(t)}{\phi^{\prime}(t)}\right)^{2}
$$

is the Schwarzian derivative, $\phi \in \operatorname{Diff}^{3}([0,2 \pi])$, and $\phi^{\prime}(0)=\phi^{\prime}(2 \pi)$.

It is convenient to rewrite the action in the form

$$
I=-\frac{1}{\sigma^{2}} \int_{0}^{1}\left[\mathcal{S}_{\varphi}(t)+2 \pi^{2} \dot{\varphi}^{2}(t)\right] d t
$$

where

$$
\begin{gathered}
\sigma=\sqrt{2 \pi} g, \quad t=\frac{1}{2 \pi} \tau, \quad \varphi(t)=\frac{1}{2 \pi} \phi(\tau), \\
\varphi \in \operatorname{Diff}^{3}([0,1]), \quad \dot{\varphi}(0)=\dot{\varphi}(1) .
\end{gathered}
$$

The functional integral for the partition function

$$
Z(g)=\int_{\dot{\varphi}(0)=\dot{\varphi}(1)} \exp \{-I\} d \varphi
$$

diverges [1]. However, as we will see later on (eq. (17)), the integral

$$
\begin{gathered}
Z_{\alpha}(g) \\
=\int_{\operatorname{Diff}^{3}([0,1])} \exp \{-I\} \exp \left\{\frac{-2\left[\pi^{2}-\alpha^{2}\right]}{\sigma^{2}} \int_{0}^{1} \dot{\varphi}^{2}(t) d t\right\} d \varphi
\end{gathered}
$$

$$
=\int_{\dot{\varphi}(0)=\dot{\varphi}(1)} \exp \left\{\frac{1}{\sigma^{2}} \int_{0}^{1}\left[\mathcal{S}_{\varphi}(t)+2 \alpha^{2} \dot{\varphi}^{2}(t)\right] d t\right\} d \varphi
$$

converges for $0 \leq \alpha<\pi$. Therefore, let us evaluate the integral (5) first.

The measure

$$
\mu_{\sigma}(X)=\int_{X} \exp \left\{\frac{1}{\sigma^{2}} \int_{0}^{1} \mathcal{S}_{\varphi}(t) d t\right\} d \varphi
$$

is quasi-invariant, and the Radon - Nikodym derivative of the measure is [6], 7]

$$
\frac{d \mu_{\sigma}^{f}}{d \mu_{\sigma}}(\varphi)=\frac{1}{\sqrt{\dot{f}(0) \dot{f}(1)}}
$$

$\times \exp \left\{\frac{1}{\sigma^{2}}\left[\frac{\ddot{f}(0)}{\dot{f}(0)} \dot{\varphi}(0)-\frac{\ddot{f}(1)}{\dot{f}(1)} \dot{\varphi}(1)\right]+\frac{1}{\sigma^{2}} \int_{0}^{1} \mathcal{S}_{f}(\varphi(t)) \dot{\varphi}^{2} d t\right\}$,

where

$$
\mu_{\sigma}^{f}(X)=\mu_{\sigma}(f \circ X) .
$$

Here, we have used the well known property of the Schwarzian derivative:

$$
\mathcal{S}_{f \circ \varphi}(t)=\mathcal{S}_{f}(\varphi(t)) \dot{\varphi}^{2}(t)+\mathcal{S}_{\varphi}(t), \quad(f \circ \varphi)(t)=f(\varphi(t)) .
$$

Take the function $f$ to be

$$
f(t)=\frac{1}{2}\left[\frac{1}{\tan \frac{\alpha}{2}} \tan \left(\alpha\left(t-\frac{1}{2}\right)\right)+1\right] .
$$

In this case,

$$
\begin{gathered}
\mathcal{S}_{f}(t)=2 \alpha^{2}, \quad \dot{f}(0)=\dot{f}(1)=\frac{\alpha}{\sin \alpha}, \\
-\frac{\ddot{f}(0)}{\dot{f}(0)}=\frac{\ddot{f}(1)}{\dot{f}(1)}=2 \alpha \tan \frac{\alpha}{2} .
\end{gathered}
$$


Now we have the following functional integrals equality:

$$
\begin{gathered}
\frac{\alpha}{\sin \alpha} \int_{\dot{\varphi}(0)=\dot{\varphi}(1)} F(\varphi) \mu_{\sigma}(d \varphi) \\
=\int_{\dot{\varphi}(0)=\dot{\varphi}(1)} F(f(\varphi)) \exp \left\{-\frac{4 \alpha}{\sigma^{2}} \tan \frac{\alpha}{2} \dot{\varphi}(0)\right\} \\
\times \exp \left\{\frac{1}{\sigma^{2}} \int_{0}^{1}\left[\mathcal{S}_{\varphi}(t)+2 \alpha^{2} \dot{\varphi}^{2}(t)\right] d t\right\} d \varphi .
\end{gathered}
$$

The next step is the choice of the function $F$. Let it be

$$
F(f(\varphi))=\exp \left\{\frac{4 \alpha}{\sigma^{2}} \tan \frac{\alpha}{2} \dot{\varphi}(0)\right\} .
$$

To find $F(\varphi)$ from the previous equation, note that for $u(t)=f(\varphi(t))$

$$
\dot{\varphi}(0)=\frac{1}{\dot{f}(0)} \dot{u}(0)
$$

Then

$$
F(u)=\exp \left\{\frac{8 \sin ^{2} \frac{\alpha}{2}}{\sigma^{2}} \dot{u}(0)\right\} .
$$

Thus for the regularized partition function we have

$$
\begin{gathered}
Z_{\alpha}(g) \\
=\frac{\alpha}{\sin \alpha} \int_{\dot{\varphi}(0)=\dot{\varphi}(1)} \exp \left\{\frac{8 \sin ^{2} \frac{\alpha}{2}}{\sigma^{2}} \dot{\varphi}(0)\right\} \mu_{\sigma}(d \varphi) .
\end{gathered}
$$

Under the substitution

$$
\varphi(t)=\frac{\int_{0}^{t} \exp \{\xi(\eta)\} d \eta}{\int_{0}^{1} \exp \{\xi(\eta)\} d \eta}
$$

the measure $\mu_{\sigma}(d \varphi)$ turns into the Wiener measure [6], 7]

$$
w_{\sigma}(d \xi)=\exp \left\{-\frac{1}{2 \sigma^{2}} \int_{0}^{1} \dot{\xi}^{2}(t) d t\right\} d \xi
$$

In this case,

$$
\xi(t)=\ln \dot{\varphi}(t)-\ln \dot{\varphi}(0), \quad \xi \in C([0,1]),
$$

and $\xi(0)=\xi(1)=0$.
Now $Z_{\alpha}(g)$ is written as

$$
\begin{gathered}
Z_{\alpha}(g)=\frac{\alpha}{\sin \alpha} \\
\times \int_{\xi(0)=\xi(1)=0} \exp \left\{\frac{8 \sin ^{2} \frac{\alpha}{2}}{\sigma^{2}} \frac{1}{\int_{0}^{1} \exp \{\xi(\eta)\} d \eta}\right\} w_{\sigma}(d \xi) .
\end{gathered}
$$

The singularity at $\alpha=\pi$ is canceled out in the ratio

$$
\frac{Z_{\alpha}(g)}{Z_{\alpha}(1)}
$$

and we can remove the regularization there

$$
\frac{Z(g)}{Z(1)}=\lim _{\alpha \rightarrow \pi-0} \frac{Z_{\alpha}(g)}{Z_{\alpha}(1)} .
$$

To evaluate the integral

$$
\int_{\xi(0)=\xi(1)=0} \exp \left\{\frac{8}{\sigma^{2}} \frac{1}{\int_{0}^{1} \exp \{\xi(\eta)\} d \eta}\right\} w_{\sigma}(d \xi),
$$

we use the following equation:

$$
\begin{gathered}
\int_{\xi(0)=\xi(1)=0} \exp \left\{\frac{-2 \beta^{2}}{\sigma^{2}(\beta+1)} \frac{1}{\int_{0}^{1} \exp \{\xi(\eta)\} d \eta}\right\} w_{\sigma}(d \xi) \\
=\frac{1}{\sqrt{2 \pi} \sigma} \exp \left\{-\frac{4(\ln (\beta+1))^{2}}{2 \sigma^{2}}\right\}
\end{gathered}
$$

The proof of the more general formula will be given in the forthcoming paper (also, see [7]). For the integral (19)

$$
(\beta+1)=-1
$$

Thus the final result is

$$
\frac{Z(g)}{Z(1)}=\frac{\exp \{-\pi\}}{g} \exp \left\{\frac{\pi}{g^{2}}\right\} .
$$

It is interesting to compare the one-loop results for $Z(g)$ in [1] with the eq. (21). Note that the power of the constant $g$ in the denominator is determined by the number of gauge fixing conditions. The one-loop result for the orbit $\operatorname{Diff}\left(S^{1}\right) / U(1)$ (eq. (3.45) in [1]) has the same form as our exact result (21).

Unlike its compact subgroup $U(1)$, the group $S L(2, \mathbf{R})$ is noncompact. Therefore, integrating over the quotient 
space $\operatorname{Dif} f^{3}([0,1]) / S L(2, \mathbf{R})$ we get the finite result for the partition function in the Schwarzian theory.

We define the Schwarzian partition function as a limit

$$
Z_{S c h w}(g)=\lim _{\alpha \rightarrow \pi-0} \frac{Z_{\alpha}(g)}{V_{\alpha}(g)} .
$$

Here, $Z_{\alpha}(g)$ is given by the eqs. (5), (17), and $V_{\alpha}(g)$ is the regularized volume of the group $S L(2, \mathbf{R})$

$$
V_{\alpha}(g)=\int_{S L(2, \mathbf{R})} \exp \left\{\frac{-2\left[\pi^{2}-\alpha^{2}\right]}{\sigma^{2}} \int_{0}^{1} \dot{\varphi}^{2}(t) d t\right\} d \mu_{H} .
$$

Note that the functional measure in the eq. (5) and the Haar measure $d \mu_{H}$ on the group $S L(2, \mathbf{R})$ in the eq. (23) are regularized in the same manner.

To perform the integration over the group $S L(2, \mathbf{R})$ in the eq. (23) we choose the representation 8]

$$
\varphi_{z}(t)=-\frac{i}{2 \pi} \ln \frac{e^{i 2 \pi t}+z}{\bar{z} e^{i 2 \pi t}+1}, \quad z=\rho e^{i \theta}, \quad \rho<1 .
$$

In this case, the Haar measure is [8]

$$
\mu_{H}(d z)=\frac{4 \rho d \rho d \theta}{\left(1-\rho^{2}\right)^{2}}
$$

The integral

$$
\int_{0}^{1} \dot{\varphi}_{z}^{2}(t) d t=\int_{0}^{1} \frac{\left(1-|z|^{2}\right)^{2} d t}{\left(e^{i 2 \pi t}+z\right)^{2}\left(e^{-i 2 \pi t}+\bar{z}\right)^{2}}=\frac{1+\rho^{2}}{1-\rho^{2}}
$$

does not depend on $\theta$. And the regularized volume of the group has the form

$$
V_{\alpha}(g)=\int_{0}^{1} \exp \left\{-\frac{\left[\pi^{2}-\alpha^{2}\right]}{\pi g^{2}} \frac{\left(1+\rho^{2}\right)}{\left(1-\rho^{2}\right)}\right\} \frac{8 \pi \rho d \rho}{\left(1-\rho^{2}\right)^{2}}
$$

$$
=\exp \left\{-\frac{\left[\pi^{2}-\alpha^{2}\right]}{\pi g^{2}}\right\} \frac{4 \pi^{2} g^{2}}{\left[\pi^{2}-\alpha^{2}\right]} .
$$

Thus we can evaluate the Schwarzian partition function

$$
Z_{S c h w}(g)=\frac{1}{4 \pi g^{3}} \exp \left\{\frac{\pi}{g^{2}}\right\} .
$$

Note that the one-loop result in [1], 2] has the same form as the exact partition function (28) obtained by the direct functional integration.

* vvbelokurov@yandex.ru

$\dagger$ shavgulidze@bk.ru

[1] D. Stanford and E. Witten, "Fermionic Localization of the Schwazian Theory", arXiv:1703.04612 1 [hep-th].

[2] J. Maldacena and D. Stanford, "Remarks on the SachdevYe-Kitaev model", Phys. Rev. D 94, 106002 (2016), arXiv:1604.07818 [hep-th].

[3] D. Bagrets, A. Altland and A. Kamenev, "SachdevYe-Kitaev Model as Liouville Quantum Mechanics", Nucl.Phys. B 911, 191 (2016), arXiv:1607.00694 [condmat.str-el].

[4] D. J. Gross and V. Rosenhaus, "A Generalization of Sachdev-Ye-Kitaev", JHEP 02, 093 (2017), arXiv:1610.01569 [hep-th].

[5] G. Mandal, P. Nayak and S. R. Wadia, "Coadjoint orbit action of Virasoro group and two-dimentional quantum gravity dual SYK/tensor models", arXiv:1702.04266v2 [hep-th].

[6] E.T. Shavgulidze, "Some properties of quasi-invariant measures on groups of diffeomorphisms of the circle", Russ. J. Math. Phys. 7, 464 (2000).

[7] V.V. Belokurov and E.T. Shavgulidze, "Extraordinary Properties of Functional Integrals and Groups of Diffeomorphisms", Phys. of Part. and Nuclei, 48, 267 (2017) [Fiz. Elem. Chastits At. Yadra 48, 194 (2017)].

[8] S. Lang, "SL $(\mathbf{R}) "$. Addison-Wesley Publishing. 1975. 